\title{
Productivité, valeur pastorale et capacité de charge des parcours naturels de la région de Maradi, Niger
}

\author{
Ali ALHASSANE ${ }^{1 *}$, Idrissa SOUMANA ${ }^{1}$, Issa CHAIBOU², Saley KARIM², \\ Ali MAHAMANE ${ }^{3}$ et Mahamane SAADOU ${ }^{3}$ \\ ${ }^{1}$ Institut National de la Recherche Agronomique du Niger, BP : 429, Niamey, Niger. \\ ${ }^{2}$ Université Dan Dicko Dankoulodo de Maradi, BP 465 Maradi, Niger. \\ ${ }^{3}$ Université Abdou Moumouni de Niamey, BP 10662 Niamey, Niger. \\ *Auteur correspondant ; E-mail: ali_alhassane@yahoo.fr ; Tel: 0022796529274.
}

\section{REMERCIEMENTS}

Aux termes de ce travail, les auteurs expriment leur gratitude au PPAAO-Niger (Programme de Productivité Agricole en Afrique de l'Ouest) et au CNS/EL (Centre National de Spécialisation en Elevage) pour le financement de toutes les activités qui ont permis la réalisation de ce travail.

\section{RESUME}

La région de Maradi dispose d'importantes ressources animales et pastorales. Mais l'extension des terres agricoles et l'accroissement du cheptel entrainent une forte pression sur les pâturages, ressources essentielles pour l'élevage au Sahel. Ainsi, cette étude vise à connaitre l'état actuel de ces pâturages en les caractérisant en termes de valeur pastorale, de productivité et de capacité de charge. Les données ont été collectées par la méthode linéaire de Daget et Poissonet (1971) et la méthode de récolte intégrale de biomasse. Les résultats montrent que la valeur pastorale des pâturages augmente avec le gradient climatique Sud-Nord tandis que la productivité des pâturages, elle, est plus élevée au Sud et plus faible au Centre de la région. La capacité de charge de tous les pâturages est très faible mais très variable entre les zones bioclimatiques, les pâturages du Sud présentant les plus grandes capacités de charge suivis des pâturages du Nord. Ainsi, la faible capacité de charge, l'extension des cultures au détriment des pâturages et l'accroissement du cheptel vont accentuer la dégradation de ces écosystèmes sahéliens déjà fragilisés ce qui aura une conséquence néfaste sur les performances zootechniques du cheptel et l'économie de la Région.

(C) 2018 International Formulae Group. All rights reserved.

Mots clés: Parcours naturels, gradient climatique, valeur pastorale, capacité de charge, Maradi, Sahel.

\section{Productivity, pastoral value and carrying capacity of rangeland in the Maradi region, Niger}

\begin{abstract}
The region of Maradi has important animal and pastoral resources. But the extension of agricultural lands and the increase in livestock are putting a lot of pressure on pastures, which are essential breeding resources in the Sahel region. The region of Maradi has important animal and pastoral resources. But the extension of agricultural land and the increase in livestock are putting a lot of pressure on pastures, which are essential
\end{abstract}


breeding resources in the Sahel. Thus, this study aims at finding out the current state of these pastures by characterizing them in terms of pastoral value, productivity and carrying capacity. The data were collected by the linear method of Daget and Poissonet (1971) and the method of integral harvesting of biomass. The results show that the pastoral value of pastures increases with the South-North climatic gradient while pasture productivity is higher in the South and lower in the Center of the region. The carrying capacity of all pastures is very low but varies a lot across bioclimatic zones, the pastures of the south having the greatest load capacities followed by pastures of the North. Thus, the low carrying capacity, cropland expansion into grasslands and the increase in livestock will intensify the degradation of these already weakened Sahelian ecosystems, which will have a detrimental effect on the zootechnical performance of the livestock and the economy of the region.

(C) 2018 International Formulae Group. All rights reserved.

Key words: Rangeland, climatic gradient, pastoral value, carrying capacity, Maradi, Sahel.

\section{INTRODUCTION}

L'élevage pastoral joue un rôle prépondérant dans l'économie des pays de l'Afrique tropicale (Lesse, 2015). Au Niger, pays sahélien à vocation essentiellement agropastorale, l'élevage constitue l'un des piliers essentiels de l'économie car il contribue à plus de $11 \%$ dans la constitution du PIB national et à plus de $25 \%$ du budget des ménages (SDDEL, 2013). La région de Maradi, située au Centre Sud du Pays est caractérisée par un climat de type sahélien semi-aride avec un gradient pluviométrique diminuant du Sud au Nord. Cette région dispose d'une vaste zone pastorale de 2455693 ha et des enclaves pastorales et espaces forestiers estimés à 149522 ha (Rép. Niger, 2013). L'élevage occupe une place importante dans l'économie de la région qui occupe la troisième place nationale avec un cheptel estimé en 2014 à 6668450 têtes de bétail toutes espèces confondues soit $16 \%$ de l'effectif national (SDDEL, 2013 ; INS, 2015). Mais durant ces dernières décennies, les parcours sahéliens connaissent de plus en plus une augmentation de la pression animale du fait d'une augmentation considérable du cheptel et d'une réduction substantielle des aires de pâturages et aires protégées qui ont régressé de $13 \%$ en 50 ans au profit des aires de cultures multipliées par 2,5. (Soumana, 2011 ; FAO et CILSS, 2012). Ainsi, dans la région de Maradi, le seuil de saturation foncière est presque atteint et les taux d'occupation des terres par l'agriculture varient de 25,61 à $56,42 \%$ ce qui induit des impacts notables sur les ressources foncières et naturelles (Rép. Niger,
2013). Or, l'accroissement du cheptel et la réduction des espaces pastoraux créent une surcharge animale qui, combinée à un surpâturage surtout en période de végétation active, entraine la dégradation de ces espaces sahéliens déjà confrontés à des sévères épisodes de sécheresse (Bourbouze et al., 2001 ; Hiernaux et Le Houérou, 2006) car pour maintenir un écosystème de parcours sain et productif, la charge ne doit pas dépasser la capacité de charge de la terre (Fouad, 2015). Ainsi, les pâturages et leur gestion constituent un défi majeur en zone sahélienne (Bechir et Mopaté, 2015) et dans la région de Maradi, la problématique pastorale constitue une des préoccupations de développement (Rép. Niger, 2013). L'étude sur la flore et la végétation des parcours de la région de Maradi (Alhassane et al., 2017) a permis de faire la typologie des pâturages; ainsi, la présente étude vise à caractériser ces différents pâturages. Spécifiquement, cette étude vise à déterminer la valeur pastorale, la productivité et la capacité de charge de chaque type de pâturage identifié suivant le gradient climatique Sud-Nord.

\section{MATERIEL ET METHODES Zone d'étude}

Cette étude a été conduite sur les parcours naturels de la région de Maradi située au Centre-Sud du Niger entre $13^{\circ}$ et $15^{\circ} 26^{\prime}$ de latitude Nord et $6^{\circ} 16^{\prime}$ et $8^{\circ} 36^{\prime}$ de longitude Est (Figure 1). Cette région est caractérisée par un gradient pluviométrique diminuant du Sud au Nord. Elle est traversée par trois compartiments phytogéographiques du Sud au 
Nord à savoir le compartiment Nord-soudanien à l'extrême Sud, le compartiment SudSahélien au Centre et le compartiment Nordsahélien au Nord (Figure 2) (Saadou, 1990). La pluviométrie moyenne annuelle des dix dernières années est de 493,3 $\pm 60,85 \mathrm{~mm}$ au Sud de la région (Station pluviométrique de Madarounfa) en bioclimat Nord-Soudanien et de 342,7 \pm 70,07 $\mathrm{mm}$ au Nord (Station pluviométrique de Fako) en bioclimat NordSahélien.

Dans la région, on distingue du point de vue pédologique six types de sols qui se sont formés en majorité sur des matériaux d'apport par les vents et les ruissellements. Les sols ferrugineux tropicaux $(70 \%$ des sols $)$, subdivisés en sols ferrugineux tropicaux peu lessivés $(5 \%)$ et sols ferrugineux tropicaux lessivés $(65 \%)$; les sols subarides brun-rouges (17\%) localisés exclusivement au Nord du Département de Dakoro; les lithosols $(0,04 \%)$; les régosols sur des roches qui n'ont pas encore subi ou ont peu subi l'évolution pédologique et les sols hydromorphes dont les caractéristiques sont dues à une évolution dominée par l'effet d'excès d'eau en raison d'un engorgement temporaire ou permanent d'une partie ou de la totalité du profil (Barké et al., 2015).

La population de la Région a été estimée à 3.404.645 habitants en 2013 avec un taux d'accroissement de 3,7\%, l'un des plus élevés du pays (INS, 2013). La forte croissance démographique a entrainé une augmentation des besoins en terres cultivables d'où la forte pression sur les ressources pastorales. C'est ainsi que les enclaves pastorales et les couloirs de passage réservés par la population à la divagation des animaux dans les parties Sud et Centre de la région sont constamment grignotés par les agriculteurs, il en est de même pour la zone pastorale située au Nord, exemptée de toute culture par la Loi $\mathrm{n}^{\circ} 61.05$ du 27 mai 1961 fixant la limite Nord des cultures.

\section{Méthodes d'études}

L'étude sur la flore et la végétation des pâturages de la région de Maradi (Alhassane et al., 2017) a permis de dresser la liste floristique et de faire la typologie de ces pâturages. Ainsi, au total dix groupements végétaux correspondant aux types de pâturages ont été identifiés comme suit. Trois groupements végétaux en bioclimat Nord-soudanien: G1 : groupement végétal à Combretum micranthum-Tripogon minimus (89 espèces, savane arbustive dense sur des bas-fonds, dépressions ouvertes ou des plateaux), G2 :groupement végétal à Zornia glochidiataSetaria pallide-fusca (65 espèces, steppe herbeuse très basse parsemée de ligneux sur des dépressions ouvertes) et G3 : groupement végétal à Digitaria horizontalis - Ipomoea vagans (73 espèces, steppe herbeuse haute dominée par sida cordifolia L. sur des dunes et glacis dégradés et sur-pâturés essentiellement sur les couloirs de passage). Quatre groupements végétaux en bioclimat Sudsahélien : G4 : groupement végétal à Acacia tortilis-Commelina nigritana (72 espèces, steppe herbeuse établie sur des dunes et des pentes); G5 : groupement végétal à Guiera senegalensis-Phyllanthus pentandrus (56 espèces, steppe herbeuse sur des bas-fonds et dépressions ouvertes); G6: groupement végétal à Alysicarpus ovalifolius-Cenchrus biflorus (66 espèces, steppes herbeuses plus ou moins arbustives sur des dunes et pentes) et G7 : groupement végétal à Sclerocarya birreaBrachiaria xantholeuca (36 espèces , steppe arborée, vallée sèche du Goulbin Kaba). Trois groupement végétaux en bioclimat Nordsahélien : G8 : groupement végétal à Maerua crassifolia-Aristida mutabilis (41 espèces, steppe herbeuse sur des dunes et pentes); G9 : groupement végétal à Acacia senegalEragrostis tremula (41 espèces, steppe arbustive sur des bas-fonds) et G10: groupement végétal à Aristida adscensionisBrachiaria villosa (59 espèces, steppe herbeuse sur des dunes et pentes) (Alhassane et al., 2017). Ainsi, la présente étude vise à caractériser la valeur pastorale, la productivité et la capacité de charge de ces différents pâturages identifiés.

\section{Collecte des données}

Les données ont été collectées en même temps et dans les mêmes placettes que celles de l'étude sur la flore et la végétation des parcours 
de la région (Alhassane et al 2017). Dans chaque placette, la strate herbacée a été relevée par la méthode linéaire de Daget et Poissonet (1971) le long de 4 lignes à l'aide d'une ficelle de $10 \mathrm{~m}$ de long, graduée tous les $20 \mathrm{~cm}$. La première ligne est placée à $5 \mathrm{~cm}$ du bord de la placette pour éviter l'effet de bordure. L'équidistance entre 2 lignes est de $10 \mathrm{~m}$. Les lignes sont disposées parallèlement à la largeur de la placette.

Une enquête pastorale auprès des éleveurs a permis d'avoir une appréciation sur l'appétence des espèces et leur nom vernaculaire car en l'absence d'analyse chimique, la qualité d'un pâturage est exprimée par le calcul de la valeur pastorale (Akpo et al., 2003). Cette enquête a été complétée par une revue bibliographique. Ainsi, une échelle de classification des espèces, qui tient compte à la fois de l'appétence de l'espèce mais aussi de sa productivité (Delpech, 1960 ; Soumana, 2011) a été élaborée.

La phytomasse a été collectée par la méthode des coupes rases, technique de récolte intégrale au maximum de biomasse (Levang et Grouzis, 1980) à l'intérieur de 5 placettes de $1 \mathrm{~m}^{2}$ $(1 \mathrm{~m} \times 1 \mathrm{~m})$ disposées dans les 4 angles et au centre de la placette. Les échantillons étaient triés et répartis en graminées et autres espèces fourragères et pesés pour déterminer les poids frais. Cent grammes de chacune des deux catégories ont été ensachés et pré-séchés au soleil et ensuite séchés à l'étuve à $65^{\circ} \mathrm{C}$ pendant $72 \mathrm{~h}$ pour avoir le poids sec (Houinato, 2001).

\section{Traitement des données}

Pour le calcul de la valeur pastorale, à chaque espèce végétale est attribué un indice de qualité spécifique sur une échelle de quatre classes variant de 0 à 3 (Akpo et Grouzis, 2000 ; Akpo et al., 2002; Ngom et al., 2012) : 0 : espèce Sans Valeur Pastorale (SVP), 1 : Espèce à Faible Valeur Pastorale (FVP), 2 : espèce à Moyenne Valeur Pastorale (MVP), 3 : espèce de Bonne Valeur Pastorale (BVP). La valeur pastorale est obtenue en multipliant les contributions des diverses espèces par les indices correspondants et en additionnant les valeurs obtenues (Hireche et al., 1999). On divise enfin le tout par le nombre de classes d'indices significatifs $(I s i \neq 0)$ et exprimé sur 100 : La valeur pastorale est donc exprimée comme suit : VP $=1 / 3 \times \Sigma C$ s $\mathrm{i} \times$ Is avec Cs : Contribution spécifique et Is : Indice de qualité spécifique, 3 : nombre de classes significatives. Pour s'affranchir du problème de surestimation, la valeur pastorale est pondérée par le recouvrement global de la végétation (RGV) (Aidoud, 1983; Akpo et Grouzis, 2000, Ngom 2012). On obtient ainsi la valeur pastorale nette ou Indice global de qualité (IGQ) donnée par la formule suivante : $\mathrm{Vpn}=$ IGQ = RGV x 1/3 $\sum$ Csi $x$ Is.

La quantité de fourrage qualifié (FQ) est obtenue en pondérant la production de biomasse (PB) par l'indice global de qualité (IGQ) (Akpo et al., 2002 ; Ngom, 2012), son expression est donc $\mathrm{FQ}=\mathrm{PB} \times \mathrm{x}$ IGQ. La capacité de charge d'un parcours est la quantité de bétail que peut supporter ce pâturage et pouvoir se régénérer; le bétail devant rester en bon état d'entretien voire prendre du poids ou produire du lait ou de la viande (Akpo, 1990). Elle s'exprime en nombre d'Unités Bétail Tropical (UBT) qu'on peut faire vivre de manière durable sur le parcours et est calculée à partir de la production de fourrage qualifié. Le calcul de la capacité de charge est accompagné d'un coefficient d'utilisation. Les estimations relatives à ce coefficient sont variables selon les types de parcours et selon les auteurs. En région sahélienne, la norme généralement retenue est de $33 \%$, mais peut osciller entre $25 \%$ et $40 \%$ (Carrière, 1996 cité par Fouad, 2015). (Boudet, 1987) estime que seul le tiers du stock fourrager de début de saison sèche est consommable par le bétail. La capacité de charge est donc: CC $(\mathrm{UBT} / \mathrm{ha} / \mathrm{an})=($ Productivité $(\mathrm{KgMs} / \mathrm{ha}) \times \mathrm{U} /$ $(6,25 \times$ période d'utilisation (365jours)). Avec: Productivité (kgMs/ha) est la productivité en fourrage qualifié, $U$ correspond au coefficient d'utilisation ; 6,25 est la ration de l'UBT estimée à $6,25 \mathrm{~kg}$ MS/j. 


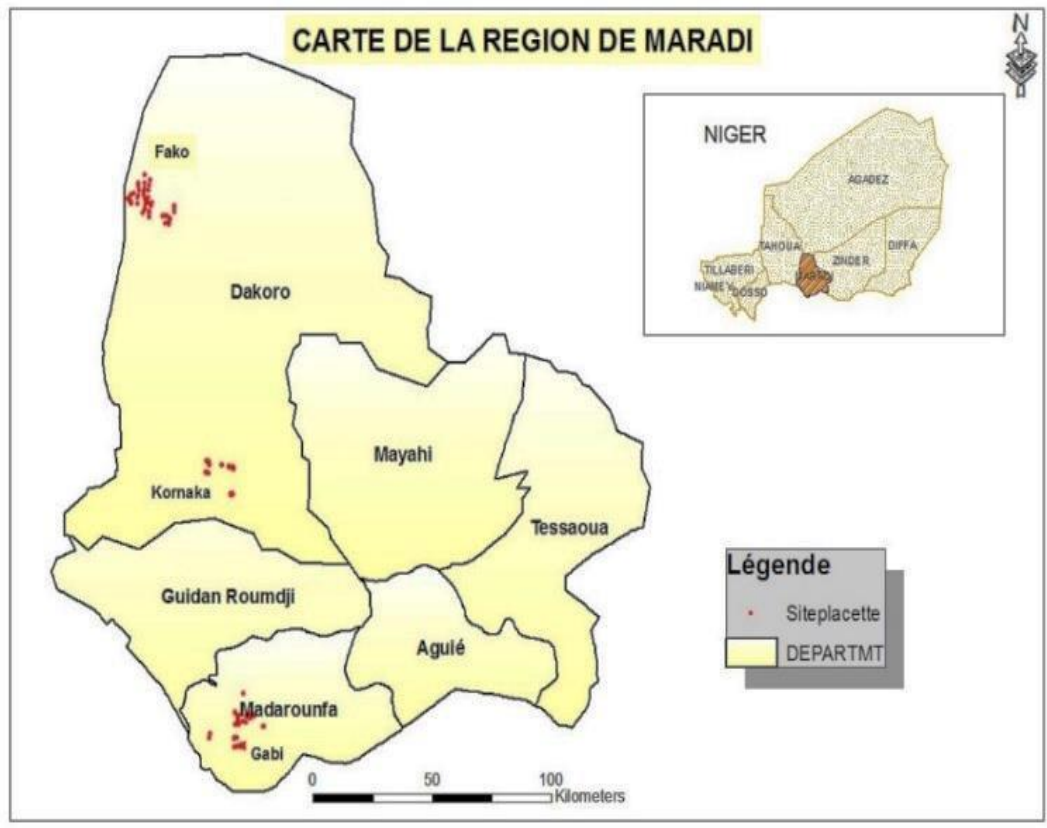

Figure 1 : Situation géographique de la région de Maradi avec les sites des relevés en points rouges.

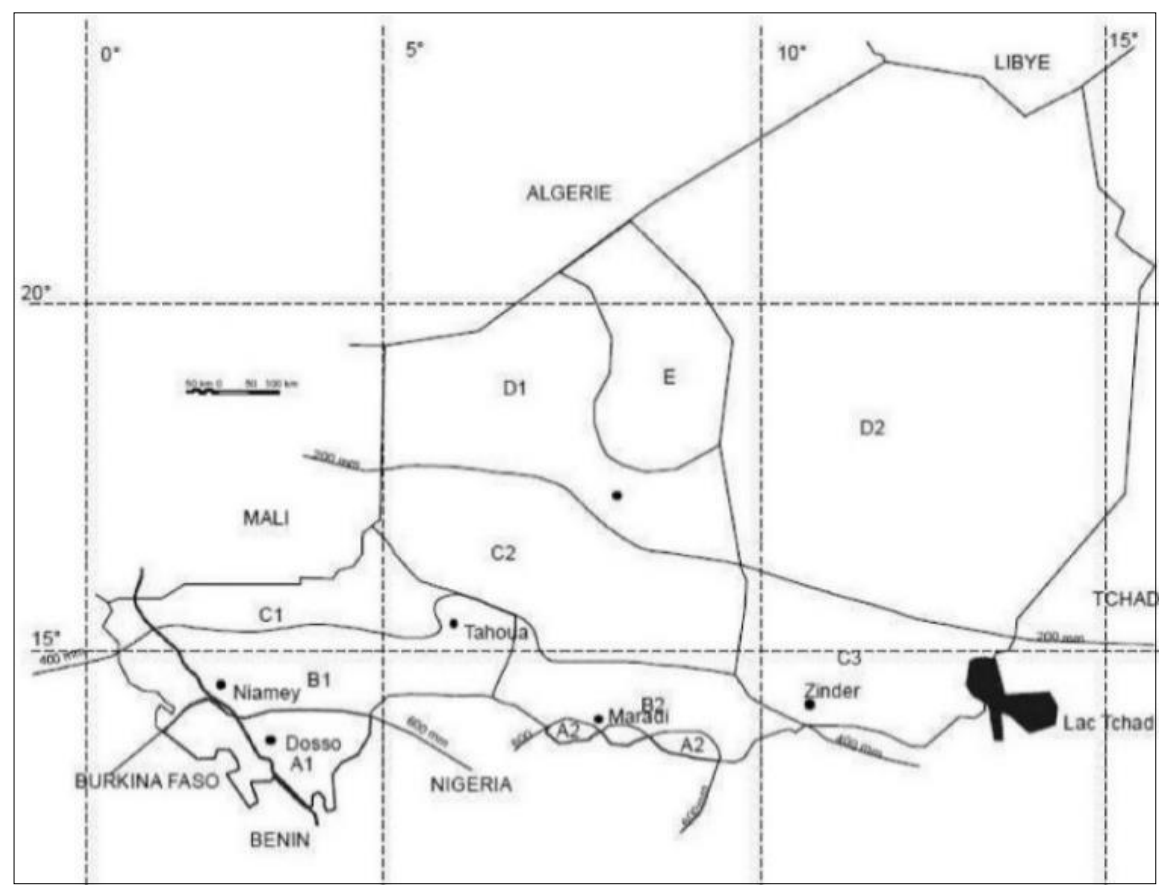

Figure 2 : Subdivision phytogéographique du Niger (Saadou, 1990). A1 : Compartiment Nordsoudanien occidental ; A2 : Compartiment Nord-soudanien central ; B1 : Compartiment Sud-sahélien occidental ; B2 : Compartiment Sud-sahélien central ; $\mathrm{Cl}$ : Compartiment Nord-sahélien occidental ; C2 : Compartiment Nord-sahélien central ; C3 : Compartiment Nord-sahélien oriental ; D1 : Compartiment Sud-saharien central ; D2 : Compartiment Sud-saharien oriental ; E : Compartiment Sud-saharien montagnard. 


\section{RESULTATS}

\section{Valeur pastorale des pâturages Spectre fourrager}

La figure 3 présente le spectre fourrager global des pâturages de la région. Sur les 135 espèces herbacées recensées, seules 12 espèces soit $8,8 \%$ sont de bonne valeur pastorale (BVP). Parmi ces espèces, 35 sont sans valeur pastorale soit $25,9 \%$. Les espèces de faible valeur pastorale (FVP) sont au nombre de 55 soit $40,74 \%$. Quant aux espèces de moyenne valeur pastorale (MVP), elles représentent $25,18 \%$ du total des espèces.

L'analyse du spectre fourrager par zone-bioclimatique (Figure 4), montre que partout les espèces de bonne valeur pastorale sont très faiblement représentées avec des proportions variant de 7 à $10 \%$. Les espèces de faible valeur pastorale sont quant à elles les plus représentées. Ainsi, en dehors des parcours du Nord, situés en zone pastorale avec moins de pression animale, les espèces de bonne valeur pastorale, les plus appétées, deviennent de plus en plus rares sur les parcours à cause de leur forte sélectivité par le bétail. Il en résulte une forte prolifération d'espèces envahissantes sans intérêt pastoral comme Sida Cordifolia L., Acanthospermum hispidum DC. et une augmentation des surfaces dénudées par endroit, très souvent encroûtées, favorisant la déplétion du sol et la matière organique.

\section{Valeur pastorale}

Aussi bien au niveau des zones bioclimatiques (Figure 5) qu'au niveau des groupements végétaux (Figure 6), la valeur pastorale varie en augmentant suivant le gradient climatique Sud-Nord. Au Sud en bioclimat Nord-soudanien, la valeur pastorale brute est de 63,29\%, inférieure à celle du Centre en bioclimat Sud-sahélien $(74,41 \%)$ et très inférieure à celle du Nord en bioclimat Nord-sahélien $(90,47 \%)$. La valeur pastorale pondérée au recouvrement aussi varie dans le même ordre passant de 56,65\% au Sud à $83,52 \%$ au Nord.

Il existe cependant des variations importantes de la valeur pastorale entre les groupements végétaux d'une même zone bioclimatique. Ainsi, au Sud en bioclimat
Nord-soudanien, la valeur pastorale brute est de $73,23 \%$ dans le groupement végétal G1 et $73,10 \%$ dans le groupement végétal G2 contre $29,75 \%$ dans le groupement végétal G3 caractérisé par un pâturage très fortement colonisé par Sida cordifolia L. Au Centre en bioclimat Sud-sahélien, les groupements végétaux G5, G6 et G7 ont des valeurs pastorales brutes comparables (respectivement $83,73 \% ; 82,13 \% ; 79,64 \%)$ par contre la valeur pastorale du groupement végétal G4 est nettement inférieure à ces valeurs $(60,88 \%)$ car colonisé par Sida cordifolia L. Au Nord par contre, la variation est peu prononcée entre les trois groupements végétaux G8, G9 et G10 (respectivement $94,38 \% ; 90,52 \% ; 87,86 \%$ ).

\section{Productivité brutes des pâturages}

Le calcul de la production brute de matière sèche par zone bioclimatique (Figure 7) montre que le Sud (bioclimat Nordsoudanien) avec 1,61 T/ha est largement plus productif que les deux autres zones. Il est suivi par le Nord (bioclimat Nord-sahélien) avec 0,93 T/ha. Le Centre (bioclimat Sud-sahélien) avec $0,54 \mathrm{~T} / \mathrm{ha}$ est le moins productif.

$\mathrm{Au}$ niveau des groupements végétaux aussi (Figure 8), la productivité brute est largement plus importante pour les groupements végétaux du Sud et moyen au Nord. Mais là aussi, des variations existent entres les groupements végétaux d'une même zone. Ainsi, au Sud le groupement végétal G3 avec 1,94 $\mathrm{T} / \mathrm{ha}$ est plus productif que les groupements végétaux G1 (1,47 T/ha) et G2 (1,47 T/ha). Cette même variation existe pour les groupements végétaux du Nord avec une productivité de $0,95 \mathrm{~T} /$ ha pour les groupements végétaux G8 et G10 contre $0,54 \mathrm{~T} / \mathrm{ha}$ pour le groupement végétal G9 le moins productif. Par contre, pour les groupements végétaux du Centre, les moins productifs, la différence de productivité est négligeable et la productivité varie de $0,54 \mathrm{~T} / \mathrm{ha}$ dans le groupement végétal G4 à 0,60 T/ha dans le groupement végétal G7.

\section{Production de fourrage qualifié}

Tout comme la productivité brute, la production de fourrage qualifié est très variable entre les trois zones bioclimatiques (Figure 9) 
et entre les groupements végétaux (Figure 10). Elle est plus importante au Sud en bioclimat Nord-soudanien et plus faible au Centre en bioclimat Sud-sahélien. Au niveau des zones bioclimatiques, la productivité est la plus élevée au Sud avec 1,06 T/ha et plus faible au Centre avec 0,45 T/ha. Le Nord présente la valeur moyenne avec 0,52 T/ha. Au niveau des groupements végétaux, au Sud les groupements végétaux G1 (1,0251 T/ha) et G2 $(1,0217 \mathrm{~T} / \mathrm{ha})$ sont les plus productifs très loin devant le groupement végétal G3 $(0,50 \mathrm{~T} / \mathrm{ha})$. Au Nord, le groupement végétal G8 (0,85 T/ha) présente la plus grande productivité en fourrage qualifié, il est suivi du groupement végétal $\mathrm{G} 10(0,77 \mathrm{~T} / \mathrm{ha})$ et $\mathrm{du}$ groupement végétal G9 (0,43 T/ha). Par conte au Centre, la productivité en fourrage qualifié des groupements végétaux est la plus basse avec peu de différence entre les groupements végétaux (G5 (0,43T/ha) ; G6 (0,45 T/ha) ; G7 $(0,37 \mathrm{~T} / \mathrm{ha})$ hormis le groupement végétal $\mathrm{G} 4$ $(0,29 \mathrm{~T} / \mathrm{ha})$ qui a une valeur relativement inferieure aux autres groupements végétaux.

\section{Capacités de charge des pâturages}

La capacité de charge de ces pâturages est très faible mais très variable entres les zones bioclimatiques (Figure 10) et entre les groupements végétaux (Figure 11). Au niveau des zones bioclimatiques, le Sud en bioclimat Nord-soudanien avec 0,16 UBT/ha présente la plus grande capacité de charge suivi par le Nord en bioclimat Nord-sahélien (0,08 UBT/ha).

Les groupements végétaux du Sud présentent les plus grandes capacités de charge, suivis des groupements végétaux du Nord. Cependant, là aussi il existe une grande variabilité entre les groupements végétaux d'une même zone bioclimatique. Ainsi, au Sud, les groupements végétaux G1 (0,149 UBT/ha) et G2 (0,149 UBT/ha) présentent les plus grandes capacités de charge très loin devant le groupement végétal G3 (0,074 UBT/ha). Au Nord, le groupement végétal G8 avec 0,124 UBT/ha présente la plus grande capacité de charge suivi du groupement végétal G10 $(0,112$ UBT/ha). Le groupement végétal G9 $(0,063$ $\mathrm{UBT} / \mathrm{ha}$ ) présente la plus faible valeur. Au Centre par contre, la capacité de charge est très faible mais très peu variée entres les groupements végétaux. En effet à part le groupement végétal G4 (0,042 UBT/ha) tous les autres groupements ont des capacités de charge similaires (G5 : 0,063 UBT/ha, G6 : 0,058 UBT/ha et G7 : 0,057 UBT/ha).

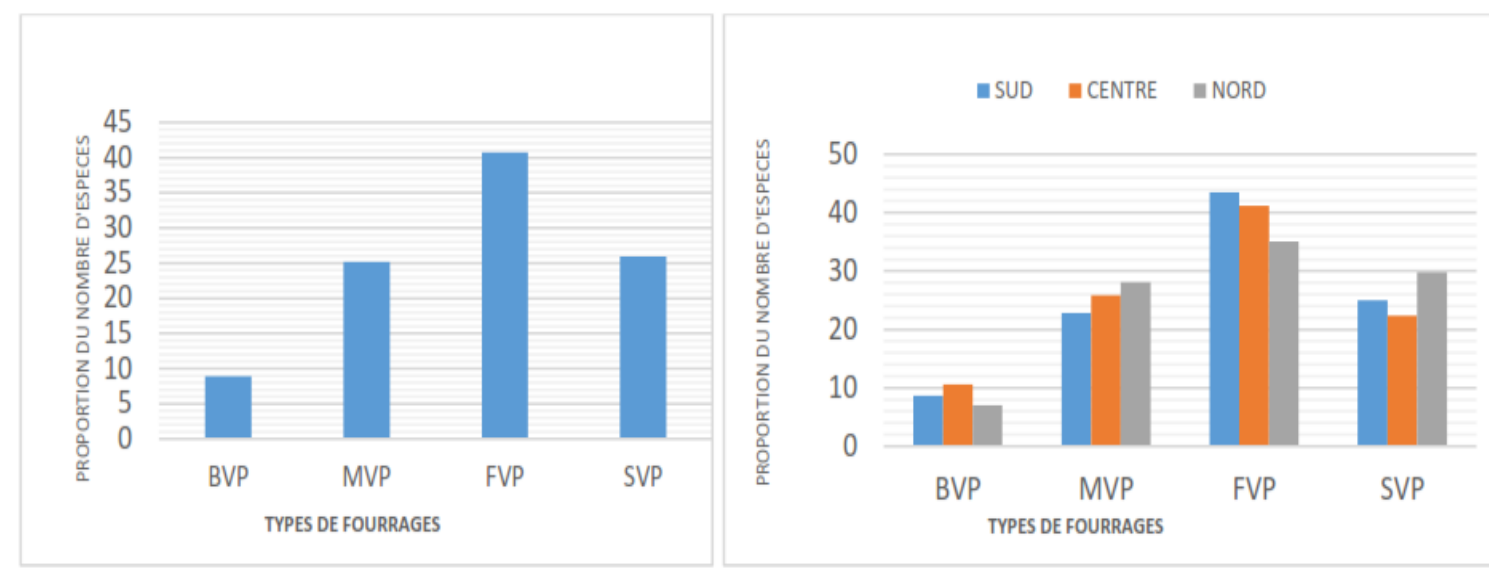

Figure 3 : Spectre fourrager global.

Figure 4 : Spectre fourrager par zone bioclimatique.

BVP : Bonne valeur pastorale ; MVP : Moyenne valeur pastorale ; FVP: Faible valeur pastorale et SVP : Sans valeur pastorale, SUD : Bioclimat Nord-soudanien, Centre : Bioclimat Sud-sahélien, Nord : Bioclimat Nord- sahélien. 


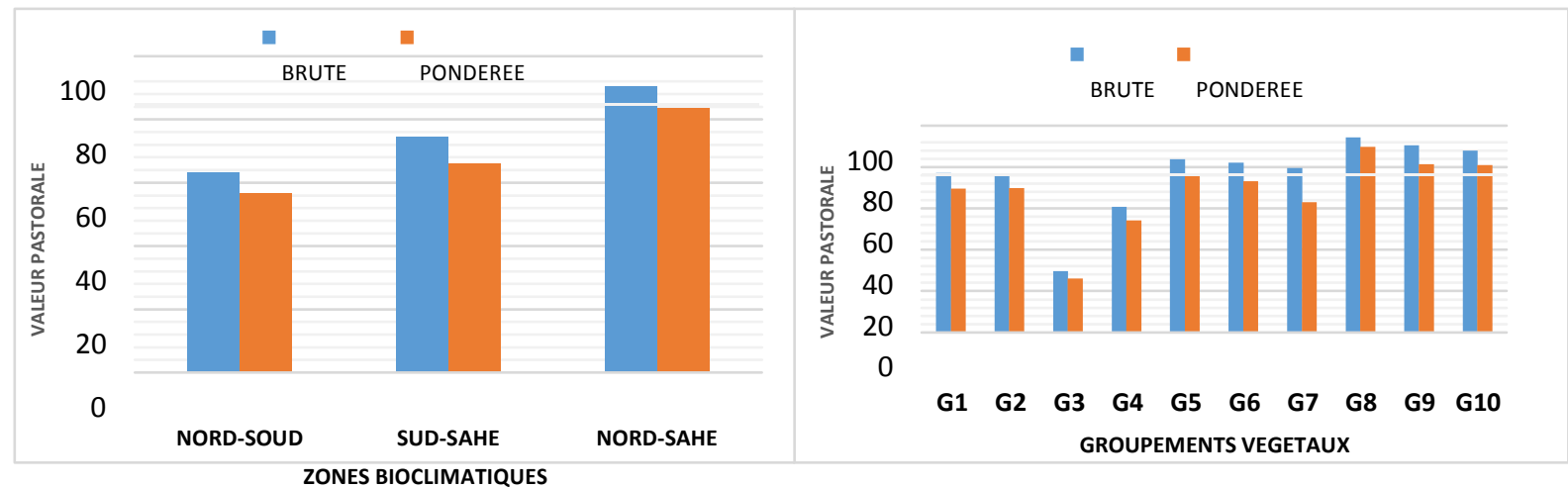

Figure 5 : Valeurs pastorales brutes et pondérées par zone bioclimatique.

Avec NORD-SOUD : Bioclimat Nord-Soudanien, SUD-SAHE : Bioclimat Sud-Sahélien, NORD-SAHE : Bioclimat Nord-Sahélien.

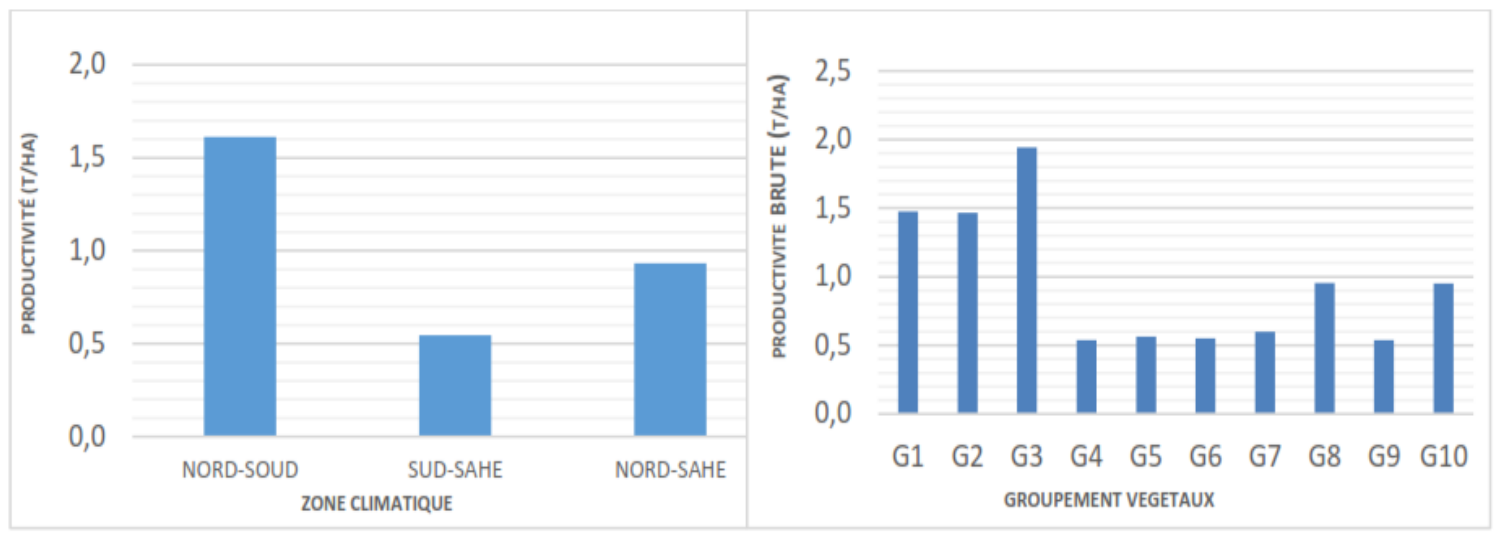

Figure 7 : Productions de matière sèche par zone bioclimatique.

Figure 8 : Production de matière sèche des différents pâturages.

Avec NORD-SOUD : Bioclimat Nord-Soudanien, SUD-SAHE : Bioclimat Sud-Sahélien, NORD-SAHE : Bioclimat Nord-Sahélien.
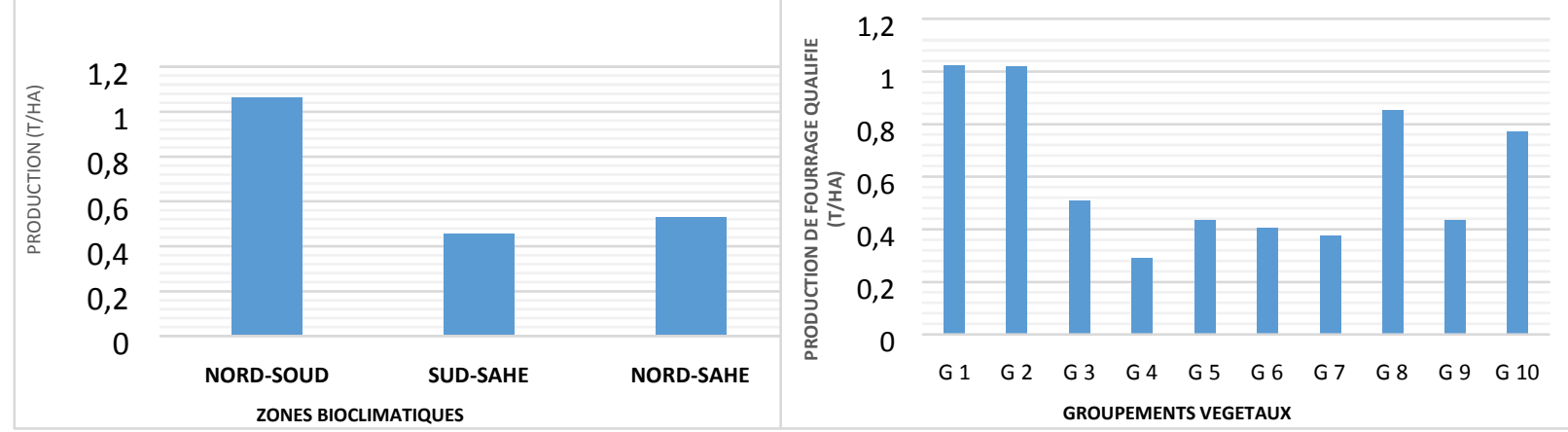

Figure 9 : Production de fourrage qualifié par zone bioclimatique.

Figure 10 : Production de fourrage qualifié des différents pâturages.

Avec NORD-SOUD: Bioclimat Nord-Soudanien, SUD-SAHE: Bioclimat Sud-Sahélien, NORD-SAHE: Bioclimat Nord-Sahélien. 


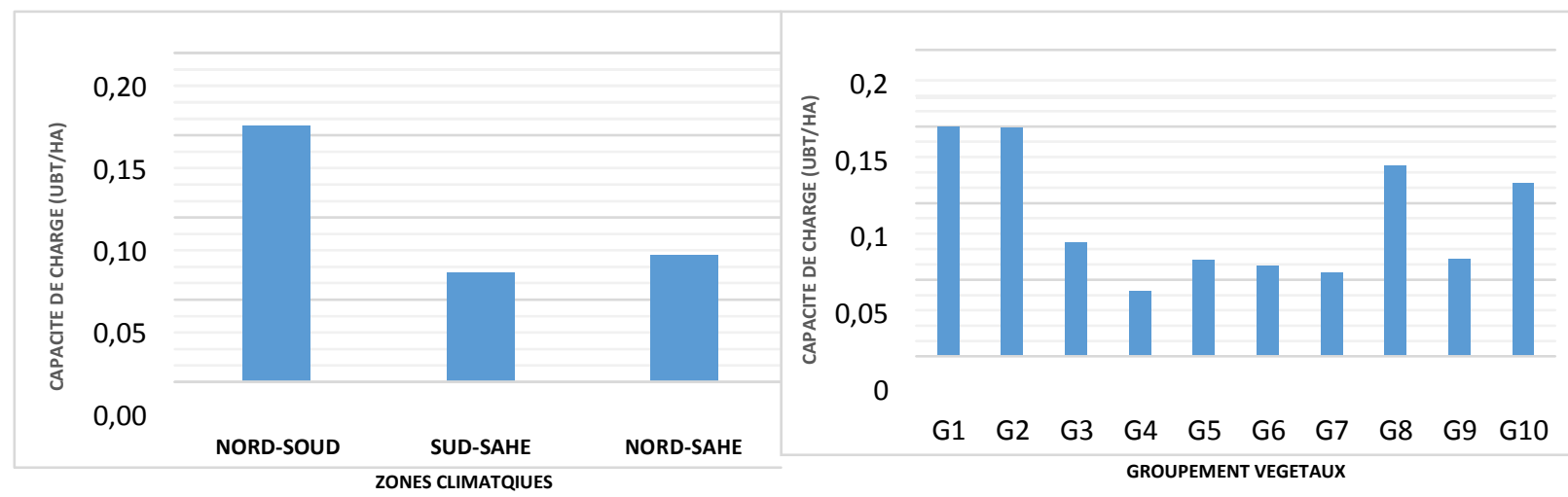

Figure 11: Capacité de charge par zone bioclimatique. Figure 12: Capacité de charge des différents pâturages. Avec NORD-SOUD : Nord-Soudanienne, SUD-SAHE : Sud-Sahélienne, NORD-SAHE : Nord-Sahélienne.

\section{DISCUSSION}

Le spectre fourrager est caractérisé par une abondance des espèces de faible ou sans valeur pastorale et une rareté des espèces de bonne valeur pastorale. Au niveau de toutes les zones bioclimatiques, les espèces de faible valeur pastorale (FVP) sont largement dominantes (De 35,08 à 43,47\%) sauf au Nord en bioclimat Nord-sahélien où les espèces de faible valeur pastorale $(35,08 \%)$ devancent de très peu les espèces sans valeur pastorale $(29,82 \%)$. Les espèces de bonne valeur pastorale sont quant à elles très peu présentes avec des proportions variant de 7,01 à $8,14 \%$ contrairement aux espèces sans valeur pastorales très présentes surtout au Nord avec des proportions variant de $22,35 \%$ à $29,82 \%$. Ces résultats sont comparables à ceux trouvés par Soumana (2011) sur les pâturages de la région voisine de cette zone d'étude (Zinder), qui a trouvé une abondance de refus (sans valeur pastorale) de $25 \%$ et d'espèces de moyenne valeur pastorale $(21 \%)$. Les espèces de grande valeur pastorale (12\%) sont les moins représentées. D'autres études réalisées sur les parcours sahéliens et Nord-soudaniens ont donné des résultats comparables (Akpo et Grouzis 2000 ; Bechir et Mopate, 2015). Par contre Akpo et al. (2002) travaillant en zone soudanienne au Senegal ont trouvé un spectre fourrager très riche en espèces de bonne valeur pastorale et très pauvres en espèces sans valeur pastorale. On pourrait donc conclure que les pâturages sahéliens sont très pauvres en espèces de bonne valeur pastorale et riches en espèces de faible valeur pastorale contrairement aux pâturages soudaniens.

Aussi bien à l'échelle des zones bioclimatiques qu'à l'échelle des pâturages, les résultats ont fait ressortir une augmentation des valeurs pastorales brutes et pondérées suivant le gradient climatique Sud-Nord. Entre les zones bioclimatiques, la valeur pastorale pondérée avec le recouvrement végétal passe de $56,7 \%$ au Sud à $83,5 \%$ au Nord. Bien qu'il existe une variabilité intra-zonale entre les différents pâturages d'une même zone, la tendance évolutive suivant le gradient climatique Sud-Nord demeure et les pâturages du Nord ont les plus grandes valeurs pastorales pondérées. Cette variation est liée à la pression sur les ressources pastorales au Sud et au Centre ce qui entraine une dégradation de ces pâturages qui entraine d'une part la disparition d'espèces de bonne valeur pastorale et leur colonisation par des espèces de très faible voire sans valeur pastorale comme Sida cordifolia L., Acanthospermum hispidum DC. et Physalis angulata $\mathrm{L}$. qui par endroit représentent plus de $80 \%$ du recouvrement herbacée et d'autre part la diminution du recouvrement du tapis herbacé $(45,25 \%$ au Sud, $62 \%$ au Centre et $91,87 \%$ au Nord). On a cependant une variation intra-zonale de cette valeur pastorale liée aux conditions locales. C'est ainsi que les pâturages du Sud en bioclimat Nord-soudanien sont globalement de moins bonne qualité que celles du Centre en bioclimat Sud-sahélien, mais le groupement végétal G4 à Acacia tortilis-Commelina nigritana du Centre très 
fortement colonisé par Sida cordifolia L. a une qualité nettement inférieure à celle des deux groupements végétaux du Sud non colonisé par cette espèce. Il en est de même du groupement végétal G7 à Sclerocarya birrea -Brachiaria xantholeuca du Centre qui malgré sa valeur pastorale brute élevée, a une valeur pastorale nette inférieure à celle des groupements végétaux G1 à Combretum micranthumTripogon minimus et G2 à Zornia glochidiataSetaria pallide-fusca du Sud à cause de son faible taux de recouvrement herbacé.

En termes de productivité, les pâturages du Sud sont les meilleurs suivis de ceux du Nord ce qui corrobore les résultats de Mahamane et al. (2009) étudiant la productivité des pâturages sahéliens qui affirment que la productivité est plus importante dans la zone Nord soudanienne que dans la zone plus septentrionale et cela est dû à la baisse de la pluviométrie. La différence de productivité entre le Nord et le Centre, pourrait s'expliquer par le fait que les pâturages du Nord se trouvent dans le ranch de Fako donc soumis à une faible pression par rapport à ceux du Centre.

Par rapport à la capacité de charge, ce sont les pâturages du Sud qui ont les plus grandes capacités de charge suivis des pâturages du Nord. Ceux du Centre ont les plus faibles capacités et cela est dû au fait que la capacité de charge est directement liée à la productivité en fourrage qualifié du pâturage or cette dernière est le produit de la production de matière sèche par la valeur pastorale nette. Donc les pâturages du Sud sont les meilleurs car ils ont une forte productivité brute pouvant compenser la faible valeur pastorale. Mais le recouvrement important des espèces sans valeur pastorale affecte la capacité de charge de certains pâturages par rapport aux autres. C'est ainsi que le groupement végétal G3 à Digitaria horizontalis-Ipomoea vagans très fortement colonisé par Sida cordifolia L. a une capacité de charge inferieure aux pâturages G10 à Aristida adscensionis - Brachiaria villosa et G8 à Maerua crassifolia - Aristida mutabilis du Nord.

\section{Conclusion}

Cette étude a révélé que la pluviométrie joue un rôle important dans la productivité des parcours sahéliens. Mais les différentes formes de dégradation des pâturages (Prolifération des espèces envahissantes, formation des plages nues) constatées surtout au sud causées essentiellement par le surpâturage et l'érosion du sol ont une influence négative sur la valeur pastorale des parcours. C'est ainsi que les zones Sud et Centre de la région, malgré la bonne pluviométrie dont elles disposent par rapport au Nord, ont des faibles valeurs pastorales dû à la colonisation des parcours par des espèces envahissantes sans valeur pastorale principalement Sida cordifolia L. et Acanthospermum hispidum DC. Mais tous les pâturages de la région ont des faibles capacités de charge ce qui, combiné à la diminution progressive des espaces pastoraux par la dégradation et la mise en culture, accentuera le surpâturage et donc la dégradation des parcours.

Ainsi, vu la faible capacité de charge de ces parcours et pour maintenir ces écosystèmes en équilibre, les mesures suivantes doivent être prises afin de garantir l'intégrité et la productivité des parcours et les performances zootechniques du cheptel : la délimitation et le balisage des espaces pastoraux et couloir de passage; la lutte contre la principale espèce envahissante des aires de pâturages du Sud et Centre de la région (Sida cordifolia L.) par le labour, l'ensemencement par des espèces de bonne valeur pastorale et la mise en défend ; la lutte contre les plages nues et les ravins formés dans les aires de pâturage par des travaux de CES/DRS et enfin, la sensibilisation de tous les acteurs impliqués la gestion et l'utilisation des espaces pastoraux sur leur fragilité, leur importance pour l'élevage et les bonnes pratiques pour une utilisation durable car une amélioration et une gestion durable du pâturage font partie des facteurs clés de la productivité du cheptel (Ousseina et al., 2013).

\section{CONFLIT D'INTERETS}

Les auteurs déclarent qu'il n'y a aucun conflit d'intérêts lié à cet article. 


\section{CONTRIBUTIONS DES AUTEURS}

L'auteur principal AA : a collecté les données, les a traitées et a rédigé le manuscrit. Les Co-auteurs IS, IC et SK : ont contribué dans le choix des sites, l'identification des espèces végétales et corrigé le manuscrit. AM et SM : ont orienté et supervisé le travail.

\section{REFERENCES}

Aidoud A. 1983. Contribution à l'étude des écosystèmes steppiques du Sud oranais : phytomasse, productivité primaire et application pastorale. Doctorat 3e cycle, USTHB, Alger, p. 253.

Akpo LE. 1990. Dynamique des systèmes écologiques sahéliens: structure spécifique, productivité et qualité des herbages : le forage de Widdu Thiengoly. Mémoire de DEA, Université Cheiq Anta Diop de Dakar, Dakar, p. 65.

Akpo LE, Grouzis M. 2000. Valeur pastorale des herbages en région soudanienne, le cas des parcours sahéliens du NordSénégal. Tropicultura, 18(1): 1-8. DOI http://horizon.documentation.ird.fr/exldoc/pleins_textes/pleins_textes_7/b_fdi_ 57-58/010024171.pdf.

Akpo LE, Masse D, Grouzis M. 2002. Durée de jachère et valeur pastorale de la végétation herbacée en zone soudanienne au Sénégal. Revue Élev. Méd. vét. Pays trop., $\quad \mathbf{5 5}(4)$ : 275-283. DOI: https://doi.org/10.19182/remvt.981 5.

Akpo LE, Banoin M, Grouzis M. 2003. Effet de l'arbre sur la production et la qualité fourragères de la végétation herbacée : bilan pastoral en milieu sahélien. Revue Élev. Méd. vét. Pays trop., 154 (10) : 619628. DOI : https://www.revmedvet.com/2003/RMV 154_619_628.pdf

Alhassane A, Soumana I, Chaibou I, Karim S, Mahamane A, Saadou M. 2017. Flore et végétation des parcours naturels de la région de Maradi, Niger. Journal of

Animal \& Plant Sciences, 34(1): 53545375.

DOI:
Barké K, Ambouta KJM, Tydjani AD. 2015. Cartographie des potentialités agricoles et forestières de la région Maradi, Colloque scientifique international « Maradi Kwalliya » sur le thème:« La coexistence intercommunautaire et la construction de la paix dans l'Histoire de la région de Maradi» du 14 au 16 décembre 2015, Maradi, Niger.

Béchir AB, Mopaté LY. 2015. Analyse de la végétation pâturée autour des ouvrages hydrauliques dans le Département du Batha Est au Tchad. Int. J. Biol. Chem. Sci., 9(3): 1557-1570. DOI : http://indexmedicus.afro.who.int

Boudet G. 1987. Connaissance et gestion de l'espace pastoral sahélien, Terroirs pastoraux et agro-pastoraux en zone tropicale, Maisons-Alfort IEMVT, étude et synthèse de l'IEMVT, 24 : 5-59.

Bourbouze A, Lhoste P, Marty A et Toutain B. 2001. Étude sur la lutte contre la désertification dans les projets de développement: Problématique des zones pastorales. p.12.

Daget P, Poissonet J, 1971. Un procédé d'estimation de la valeur pastorale des pâturages. Exposé présenté à la quatrième assemblée générale de la fédération européenne des herbages à Lausane, p.8.

Delpech R. 1960. Critère et jugement de la valeur agronomique des prairies. Fourrages, $4: 83-98$. DOI : http://afpfasso.org/index/action/page/id/33/title/Les -articles/article/141

Fouad R. 2015. Contribution à l'évaluation des ressources fourragères des parcours steppiques de l'Est Algérien « Cas de la région de Tébessa ». Doctorat d'Etat, Université El hadj Lakhdar, Batna, p.120.

Hiernaux P, Le Houérou HN. 2006. Les parcours du Sahel. Sécheresse, 17 (1-2) : 51-71. DOI : https://www.rmportal.net/framelib/parco urs-sahel.pdf. 
Hirche A, Boughani A, Nedjraoui D. 1999. About grassland quality assessment in arid areas. In: Etienne M. (ed.). Dynamics and sustainability of Mediterranean pastoral systems. Zaragoza : CIHEAM, 1999. p. 193-197.

Houinato MRB. 2001. Phytosociologie, écologie, production et capacité de charge des formations végétales pâturées dans la région des Monts Kouffé (Bénin). Thèse de doctorat, Université Libre de Bruxelles, Bruxelles, p.219.;

Institut National de la Statistique. 2013. Maradi en chiffres, Edition 2013, Institut National de la Statistique, Direction Régionale de Maradi, République du Niger, p. 2 ;

Institut National de la Statistique. 2015. Annuaire statistique régional de Maradi : 2010-2014, Edition 2015, République du Niger, p.158.

Lesse P, Houinato MRB, Djenontin J, Dossa H, Yabi B, Toko I, Tente B, Sinsin B. Transhumance en République du Bénin : états des lieux et contraintes. Int. J. Biol. Chem. Sci., 9(5): 2668-2681. DOI : http://indexmedicus.afro.who.int

Levang P, Grouzis M. 1980. Méthodes d'étude de la Biomasse herbacée des formations sahéliennes: application à la Mare d'Oursi, Haute-Volta. Acta OEcologica, OEcol. Plant., 1(15): 231-244. DOI : http://horizon.documentation.ird.fr/exldoc/pleins_textes/ pleins_textes_5/b_fdi_04-05/03922.pdf ;

Mahamane A, Saadou M, Danjimo MB, Saley K, Yacoubou B, Diouf A, Morou B, Mamane Maarouhi I, Soumana I, Tanimoune A. 2009. Biodiversité végétale au Niger : état des connaissances actuelles. Annales de l'Université de Lomé, 18 : 81-93.
Ngom D, Bakhoum A, Diatta S, Akpo LE. 2012. Qualité pastorale des ressources herbagères de la réserve de biosphère du Ferlo (Nord-Sénégal). Int. J. Biol. Chem. Sci., 6(1): 186-201. DOI: http://dx.doi.org/10.4314/ijbcs.v6i1.17

Ousseina S, Fortina R, Marichatou H, Yenikoye A. 2013. Dynamique du peuplement herbacé de la station sahélienne expérimentale de Toukounous (Filingué-Niger). Int. J. Biol. Chem. Sci., 7(2): 657-671. DOI :http://indexmedicus.afro.who.int

République du Niger. 1961. Loi n 61.05 du 27 mai 1961 fixant la limite Nord des cultures.

République du Niger. 2013. étude bilan du code rural: région de Maradi, aidemémoire, p.10.

Saadou M. 1990. La végétation des milieux drainés nigériens à l'est du fleuve Niger. Thèse de doctorat, Université de Niamey, Niamey, p. 393.

Stratégie de Développement Durable de l'Elevage 2013-2035. 2013, République du Niger, P.78.

Soumana I. 2011. Groupements végétaux pâturés des parcours de la région de Zinder et stratégies d'exploitation développées par les éleveurs Uda'en. Thèse de Doctorat, Université Abdou Moumouni de Niamey, Niamey, p. 229. 